\title{
Hitit Tanrlar Topluluğunda Bir Tanrıça: Kappariyamu
}

\author{
Nursel Aslantürk*
}

\begin{abstract}
$\ddot{O}_{z}$
Eski Anadolu'nun en önemli uygarlıklarından biri olan Hitit uygarlığı tarih boyunca çok sayıda önemli uygarlığa beşiklik etmiş olan ve uygarlıklar tarihinde birçok ilklere tanıklık eden Anadolu'nun verimli toprakları üzerinde MÖ. II. bin yıllarında kurulmuştur. Hint Avrupalı kavimlerden olan Hititler, "Hatti Ülkesi" olarak adlandırdıkları Anadolu'da, siyasi birliği kurduktan sonra yayılmacı bir politika benimsemişlerdir. Bu politika sonucunda Hitit Devleti, Güney ve Güneydoğu Anadolu, Mezopotamya ve Kuzey Suriye'de etki alanı oldukça geniş bir imparatorluk haline gelmişlerdir. Boğazköy'de yapılan arkeolojik araştırmalar sonucunda bulgulanan Hitit dilinde yazılmış olan çiviyazılı kil tabletlerden Hititler'in dine dayalı bir yönetim sistemi benimsedikleri öğrenilmektedir. Bu çalışma, politeist (çok tanrılı) bir inanç sistemine sahip olan Hititlerin resmi dininde ve pantheonunda yer alan Tanrıça Kappariijamu'nun kökenini, adının anlamını, tanrıçanın ne zaman ve nasıl kutsandığını Hitit çiviyazılı belgelerden elde edilen veriler doğrultusunda filolojik değerlendirmelerle ortaya koymayı amaçlamaktadır.
\end{abstract}

Anahtar Kelimeler: Hitit, Hatti, Kappariyamu (Kappariiamu), Kappariyamuwa (Kappariiamuua ), Din, Tanrı(ça).

\section{A Goddess in the Community of Hittite Goddess: Kappariyamu}

\begin{abstract}
The Hittites, as one of the greatest civilizations of Ancient Anatolia, had established their civilization in $2^{\text {nd }} \mathrm{BC}$ on the fertile lands of Anatolia which has been a cradle to

* Doç. Dr., Çankırı Karatekin Üniversitesi, Edebiyat Fakültesi, Arkeoloji Bölüm, Çankırı/ TÜRKIYE, n.aslanturk3@gmail.com ORCID: 0000-0003-1470-2727

DOI: 10.37879/belleten.2021.361

Makale Gönderim Tarihi: 12.09.2019 - Makale Kabul Tarihi: 05.04.2021
\end{abstract}

Belleten, Ağustos 2021, Cilt: 85/Say1: 303; 361-382 
many civilizations and has witnessed various first strings throughout history. As one of the Indo-European tribes, the Hittites established the political unity and later, they pursued an expansionist policy in Anatolia where they call "the Hattian country". As a consequence of this policy, the Hittites became an ample empire with a great influence in South and Southeast Anatolia, Mesopotamia and Northern Syria. According to the cuneiform clay tablets written in Hittite language that were discovered in result of the archeological researches in Boğazköy, it has been learned that the Hittites adopted a regime of governance based on religion. This work aims at revealing the Goddess Kappariiamu's origin, the meaning of her name and when and how the goddess was blessed in the official religion and pantheon of Hittites, who had a polytheistic belief system, based on the data provided by the Hittite cuneiform texts with philological analysis.

Keywords: Hittite, Hattian, Kappariyamu (Kappariiamu), Kappariyamuwa

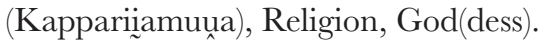

\section{Giriş}

MÖ.II.binde Anadolu'da büyük bir uygarlık kurmuş olan Hint-Avrupalı kavimlerden Hititler’in; insanoğlunun her tarihsel süreçte yaratmış olduğu din ve tanrı olgusunu sistemli bir şekilde oluşturdukları görülmektedir ${ }^{1}$. Tanrılarını insan şeklinde tasavvur eden Hititler; ancak bazı tanrıların hayvansal motifleri de söz konusudur (boğa, geyik vs.); politeist yani çok tanrılı bir inanışa sahiptiler.

Bu çalışmamızda Hitit tanrılar topluluğunun bir üyesi olan Tanrıça Kappariïamu (Kappariiamuua Na $_{\sim}$ Kappariija-A.A) Hitit çiviyazılı metinlerden elde edilen bilgiler doğrultusunda tüm yönleriyle ele alınacak ve ayrıca Tanrıça Kappariïamu'nun "Koruyucu Tanrı grubu” ve Kuškurša- ile bağlantısı değerlendirilecektir:

1 Hitit dininin tarihi ve gelişimi için bk. Yasemin Arıkan, "Hitit Dini Üzerine Bir İnceleme", $A \ddot{U}$ DTCF Dergisi, C. 38/ S.1-2, 1996, s. 271-285; Yasemin Arıkan, "Hitit Dininin Tarihi Gelişimi ve Hitit Panteonu", Yaşar Coşkun'a Sayg Yazılan, 2018, s. 41-72. 
Tanrıçanın adının geçtiği Hitit çiviyazılı metinler²:

\begin{tabular}{|c|c|c|}
\hline Abs. & ${ }^{\text {DKap-pa-ri-ia-mu-u }}$ & KUB 7.36 ay. sağ. k. 16; KUB 53.11 II 31 \\
\hline & D[Kap-pa-r]i-ia-mu-u & KUB 56.33 ay. sağ kol. 4 \\
\hline & ${ }^{\mathrm{D}}[$ Kap-pa-r]i-ia-A.A & KUB 55.54 I 30. \\
\hline \multirow[t]{2}{*}{ Akk. } & ${ }^{\mathrm{D}}$ Kap-pa-ri-ia-mu-u-u्रa-an & Bo 4801 I 10. \\
\hline & ${ }^{\text {DKap-pa-ri-ia-mu-un }}$ & KUB 20.13 I 14; KUB 55.43 II 17; IV 16. \\
\hline \multirow[t]{2}{*}{ Dat. } & ${ }^{\text {DKap-pa-ri-ia-mu-i }}$ & KUB 53.11 II 25. \\
\hline & DKap-pa-ri-ia-mu-ú-i & $\begin{array}{l}\text { KBo } 18.97 \text { 10+KBo } 21.89 \text { IV } 16 \text {; KUB } \\
53.11 \text { II } 18 \text {. }\end{array}$ \\
\hline$[(-)$ & ${ }^{\mathrm{D}}$ Kap-pa-ri-ia-mu[(-) & KBo 11.35 ay.3; KUB $20.39 \mathrm{~V} 14$. \\
\hline \multirow[t]{3}{*}{ Fr. } & ${ }^{\mathrm{D}} \mathrm{Kap}-\mathrm{p}[\mathrm{a}-$ & KUB 53.11 II 9; KUB 60.41 öy. 4 \\
\hline & ${ }^{\mathrm{D}} \mathrm{Ka}[\mathrm{p}-$ & KUB 7.36 ay. sağ kol. 4 \\
\hline & $\mathrm{D}[$ & KUB 2.3 IV 12. \\
\hline
\end{tabular}

Hitit çiviyazılı metinlerde adı geçen Kappariiamu Hatti kökenli ${ }^{3}$ bir tanrıçadır ${ }^{4}$. Genellikle festival metinlerinde geçen bu tanrıçanın adının anlamı da ne yazık ki tam olarak bilinmemektedir. Ancak "muuna-: güç, kuvvet, kudret" kelimesinin ideogram yazılışının "A.A"6 olduğu göz önünde bulundurulduğunda, kelime Hititçe birleşik olarak "kappariia-muu्रa: kappari- gücü" şeklinde yeniden kurulabilinir. Hattice karşllığının ise "kapparilili" olduğu düşünülmektedir?. Ayrıca tanrıçanın, Hitit Imparatorluk dönemi krallarından II.Muršili’nin anallerinde geçen, Tanrıça

2 Emmanuel Laroche, Recherches sur les noms des dieux hittites, (RHA VII/46) 1946-1947, s.27; Gabriella Frantz-Szabó, "Kappariiamu”, RlA 5, 1976-1980, s. 400; Ben H.L.van Gessel, Onomasticon of the Hittite Pantheon, Part I, Leiden-Newyork-Köln 1998, s. 222; 2001 yılı sonrasında yayınlanan Hitit çiviyazılı metinler ise taranmış olup; tanrıçanın adının geçtiği metinler elde edilmiştir.

3 Laroche, Recherches, s. 27; Frantz-Szabó, agm., s. 400.

4 Gregory McMahon, The Hittite State Cult of the Tutelary Deities, AS 25, Chicago 1991, s. 18; ancak Charles W. Steiler, The Solar Deities of Bronze Age Anatolia, Studies in Texts of the Early Hittite Kingdom, StBoT 62, Wiesbaden 2017, s.291 dn.909." da ise tanrı olarak tanımlamakta iken Laroche, Recherches, s.27'de; 174; Volkert Haas, Geschichte der Hethitischen Religion, HbOr I/15, Leiden 1994, s. 487'de; Frantz-Szabó, agm., s. 400'de Kappariijamu'nun cinsel kimliği üzerine kesin bir ifade kullanmamişlardır.

5 HW: 146; HHw: 108; HZL: 304; $\operatorname{HED}(\mathrm{m})$ : 196-197; CHD(l-n): 314-316; ayrıca kelimenin diğer anlamları için ve bibliyografya ile birlikte bk. Meltem-Doğan Alparslan, Hitit krah II. Muwatalli: Kişiliği ve İcraatı (Filolojik Belgeler Işı̆̆ında). İstanbul: Ege Yayınları 2018, s.3-5.

6 Anlam için bk. HZL: 304; yazılış için krş. Laroche, Recherches, s.27.

7 Laroche, age., 1947, s. 27; ayrica bk. Oğuz Soysal, Hattischer Wortschatz in hethitischer Textüberlieferung, HbOr I/74, Leiden 2004, s. 532 
Hatepinu'nun bir tapınağının olduğunu bildiğimiz ve Kaška bölgesinde yer aldığı düşünülen Kapperi kentiyle ${ }^{8}$ de bağlantılı olduğu9 ; dolayısıyla tanrıçanın adının kentin adına getirilen "muuxa" ekiyle oluşturulduğu ifade edilmektedir ${ }^{10}$. F.P.Daddi ise, "Osservazioni su alcuni teonimi hattici" adlı çalışmasında s.102'de tanrıçanın adını "kapperi-" yer adından türetilmiş olup; "-mu" eki eklenmiştir." şeklinde analiz etmektedir.

Hitit çiviyazılı belgelerde Tanrıça Kappariijamu'nun adıyla en erken Eski Hitit dönemine tarihlendirilen metinlerde karşılaşmaktayız. Huanhana'nın Kültüne (CTH 668) ait olan ve maalesef içerik açısından çok fazla bilgi vermeyen KUB 60.41(Bo 1291)(+) KUB 54.50 (Bo 1434) (+)DBH 46/2.157 (Bo 4958)+HHT 73 (Bo 5478) (+) Bo $7937^{11}$ metinde tanrıçanın adı, Tanrıça Hualmašuitta ${ }^{12}$ ve Tanrı Telipinu ile birlikte geçmektedir ${ }^{13}$. Söz konusu metnin katalog numarasını ise; D.Groddek, DBH 20, 2006'daki çalışmasında, s.41'de "CTH 647: Prens tarafindan kutlanılan festivaller" olarak verirken; S. Alp, TTKY VI/23, 1983, s.234'te ise CTH 648 olarak vermektedir.

Hitit bayramlarından, çok sayıda Hattice terim, tanrı ve tanrıça adlarının geçtiği dolayısıyla Hatti kökenli olduğu düşünülen KI.LAM (GTH 627) bayramında tanrıçanın adı Tahantiu ile birlikte geçmektedir:

8 Einar von Schuler, Die Kaškäer. Ein Beitrag zur Ethnographie des alten Kleinasien, Berlin 1965, s. 79; Hayri Ertem, Boğazköy Metinlerinde Geçen Coğrafya Adlan Dizini, Ankara 1973, s. 67; Heinrich Otten, "Kapperi", RlA 5, 1976-1980, s. 400; Giuseppe F. del Monte-Johann Tischler, Die Orts- und Gewässernamen der hethitischen Texte, RGTG VI, Wiesbaden 1978, s.175; Giuseppe F. del Monte, Die Orts- und Gewässernamen der hethitischen Texte. Supplement, RGTG 6/2 Wiesbaden 1992, s. 64.

9 Laroche, Recherches, s. 27; Emmanuel Laroche, Les noms des Hittites. Paris 1966, s. 323 dn.17; Haas, age., s. 487 dn. 166.

10 Laroche, Les noms des Hittites, s. 323 dn.17; CHD(1-n): 314.

11 Detlev Groddek, Hethitische Texte in Transkription. KUB 60, DBH 20, Wiesbaden 2006, s. 41; Ayrica bk. Erich Neu, Althethitische Ritualtexte in Umschrift, StBoT 25, Wiesbaden 1980, s. 109; Sedat Alp, Beiträge zur Erforschung des hethitischen Tempels. Kultanlagen im Lichte der Keilschrifttexte. Neue Deutungen, Ankara 1983, s. 234; Itamar Singer, The Hittite KI.LAM Festival, StBoT 27, Part: I, Wiesbaden 1983, s. 114 dn.78; Daisuke Yoshida, Untersuchungen zu den Sonnengottheiten bei den Hethitern, THeth 22, Heidelberg 1996, s. 225; Ada Taggar-Cohen, Hittite Priesthood, THeth 26, Heidelberg 2006, s. 246; Rukiye Akdoğan, Hethitische Texte Bo 4658-Bo 5000. Teil 1: Transkriptionen. Teil 2: Autographien, DBH 46, Wiesbaden.2016, s. 108-109; Steiler, age., s. 88.

12 Piotr Taracha, Religions of Second Millennium Anatolia, DBH 27, Wiesbaden, 2009, s. 30.

13 öy. $\mathbf{4}^{\prime}[$

$\left.{ }^{\mathrm{D}}\right] H a l-m a-a \check{s}-\check{s} u-i t-t u-u n{ }^{\mathrm{D}} K a p-p[a-r i-i a-m u-u n]$

D] Te-li-pí-nu-un...

Belleten, Ağustos 2021, Cilt: 85/Say1: 303; 361-382 


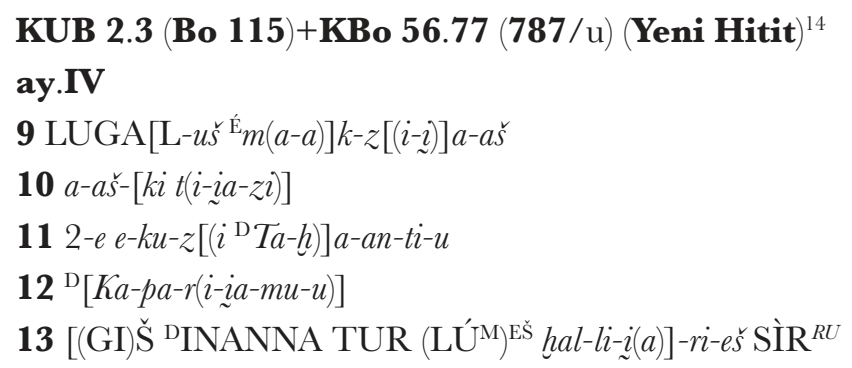

\section{Tercüme:}

$9 \mathrm{Kra}[1, \mathrm{~m}(\mathrm{a})] \mathrm{kz}[(\mathrm{ii})] \mathrm{a}$ (yapısı)nın

10 kapı[sinda(n) $\mathrm{g}$ (irer.)]

11 iki kez içe[(r: Tah)]antiu (ve)

12 [Tanrıça Kapar(iiamu)] (için)

13 [(Küçü)k lir (çalınır). (Şarkıcıla)]r şarkı söylerler.

\section{Metnin Değerlendirmesi}

Söz konusu bayrama ait metinden, kırık olmasına rağmen, Tanrıça Kappariïamu'nun kral tarafindan kutsandığı; adının ise Tahantiu'dan sonra geçtiği görülmektedir. Bu da adı geçen tanrıçanın; Tahantiu'dan sonra kutsandığını göstermektedir. Metinin ilgili kısmından adı geçen tanrılar için içildiği, seremoniye ise küçük lirin çalınıp, şarkıcıların şarkı söylemesiyle devam edildiği öğrenilmektedir. Ayrıca söz konusu metnin ay.IV 9-14; Yeni Hitit dönemine tarihlendirilen KUB 56.33(Bo 2505)(+)IBoT 4.140(Bo 1205)(+)KUB 59.36 (Bo 3087)(+)Bo 3370 ${ }^{15}$ metnin ay. $\mathrm{x}+1-7$ ile duplikattır ${ }^{16}$.

14 Itamar Singer, The Hittite KI.LAM Festival, StBoT 28, Part: II, Wiesbaden 1984, s. 68; aynca bk. Frank Starke, Untersuchungen zur Stammbuilding des kelischrift-luwischen Nomens, StBoT 31, Wiesbaden.1990, s. 401 ve dn.1446; Enrico Badalì, Strumenti musicali, musici e musica nella celebrazione delle feste ittite, THeth 14/1, Heidelberg. 1991, s. 113; Haas, age., s. 335 dn.174; 508 dn.144; 627 dn.54; Jörg Klinger, Untersuchungen zur Rekonstruktion des hattischen Kultsichicht, StBoT 37, Wiesbaden.1996, s. 241-242; Daliah Bawanypeck, Die Rituale der Auguren, THeth 25, Heidelberg 2005, s. 291; Taggar-Cohen, Hittite Priesthood, s. 338-339.

15 Metin çalışması için bk. Singer, StBoT 28, s. 68; Detlev Groddek, Hethitische Texte in Transkription. KUB 59, DBH 14, Dresden 2004, s. 64-65; Johann Tischler, Hethitische Texte in Transkription KUB 56 und KUB 57, DBH 49, Dresden 2016, s. 49-52.

16 Tischler, DBH 49, s. 49. 


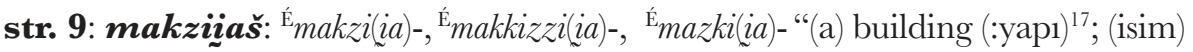
ayinlerde de kullanılan bir yap1 ${ }^{18}$; $\dot{E}_{m a k z i-}{ }^{E_{m}}$ makziia Gebäude, in dem sich u.a. das Königspaar wäscht und/oder ankleidet: bir bina, içinde krali çiftin yıkandiğı ve/ya giyindiği $)^{19}$. “9 .......... $\left.{ }^{\dot{E}} m(a-a)\right] k-z[(i-i)] a-a s ̌ \mathbf{1 0}$ $a-a s^{2}[k i t(i-i a-z i)]$ : makziiia yapısının kapısında(n) geçer/girer." ifadesiyle de yapının bir kapısı olduğu öğrenilmektedir.

makziiias: Sg.Gen.

Tanrıçanın adı, Koruyucu Tanrının av çantasının (kurša- $)^{20}$ yenilenmesi ile ilgili (GTH 683) metinlerde çok sık geçmektedir:

\section{a.KUB 20.13(Bo 2130)(Yeni Hitit $)^{21}$}

\section{öy.I}

$\mathbf{9}^{\prime}$ nu ha-an-te-ez-zi pal-šs ${ }^{\mathrm{D}}$ [UTU? TUŠ $\left.a s ̌ / G U B-a s ̌ ~ e-k u-z i\right]$

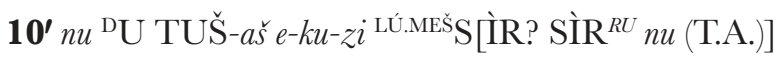

$11^{\prime}$ GUB- $a s ̌ s e-k u-z i$ LÚ.MEŠALAM.Z[U $\mathrm{U}_{9}$

12' [nu $\left.{ }^{\mathrm{D}} z\right] i-i t-h a-r i-i a-a n \mathrm{GU}\left[\mathrm{B}-a e^{2}\right.$ e-ku-zi]

13' [nu DLAMMA URU Ta-t] $a$-šu-na GU[B-aš e-ku-zi]

$\mathbf{1 4}^{\prime}\left[n u{ }^{\mathrm{D}} K a p-p a-r i-i a-m\right] u-u n$ GU[B-aš e-ku-zi]

15' $[n u$ DLAMMA URU $H a-t e-e] n-z u-u a^{\mathrm{D}}{ }^{\mathrm{D}}$ [x GUB-as']

$\mathbf{1 6}^{\prime}[e-k u-z i \quad]$

\section{Tercüme:}

9' Ve ilk kez [Güneş Tanrısı (için) oturarak/ayakta içer.]

$1 \mathbf{1 0}^{\prime}$ Fırtına Tanrısı (için) oturarak içer. Şar[kıcılar şarkı söylerler ve (Tanrı/ça x)]

11' ayakta içer. Soytarı[lar (/hokkaba[zlar)

12' [Tanrı Z]itḩariiia (için) aya[kta içer.]

$17 \mathrm{CHD}(\mathrm{l}-\mathrm{n}):$ 123; MHH: 418.

18 Ahmet Ünal, Hititçe-Türkçe, Türkçe-Hititçe Büyük Sözlük, Ankara 2016, s. 331.

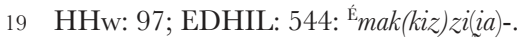

20 McMahon, age., s. 250 vd.

21 McMahon, age., s. 172; Detlev Groddek, Hethitische Texte in Transkription. KUB 20, DBH 13, Dresden 2004a, s. 24; ayrıca tercüme için de bk. McMahon, age., s. 173. 
13' [Tat]ašuna Kenti Koruyucu Tanrısı (için) ayak[ta içer.]

14' [Tanrıça Kappariiam]u (için) aya[kta içer.]

15' [Hate]nzuuua Kenti Koruyucu Tanrısı, Tanrı/ça x (için) [ayakta]

16' [içer.

Bu metinde Tanrıça Kappariijamu'nun Hitit dini metinlerinde sıkça geçen “Tanrı/ ça için içmek”"22 eylemiyle kutsandığını öğrenmekteyiz. Metinde; tanrıçanın adı koruyucu tanrılarla birlikte geçmektedir. Adı geçen tanrıça, Tatašuna Kenti Koruyucu Tanrisindan sonra, Hatenzuua Kenti Koruyucu Tanrısindan ise önce geçmektedir. Bu da tanrıçanın, Tatašuna Kenti Koruyucu Tanrısından sonra, Hatenzuưa Kenti Koruyucu Tanrısından ise önce kutsandığını göstermektedir. Söz konusu metinden tanrıçanın adının kurša- festivalinde, koruyucu tanrılarla birlikte üçlü bir grup şeklinde geçmesi Tanrıçanın; Hitit pantheonundaki "koruyucu tanrılar" grubu içinde yer aldığını düşündürtmektedir ${ }^{23}$. Ancak; Hitit çiviyazılı metinlerde geçen "DAMMA" ideogramının yalnızca "erkek koruyucu tanrıları" ifade etmesine dayanarak ${ }^{24}$ Tanrıçanın; Hitit pantheonundaki "koruyucu tanrılar" grubu içinde yer alan bir tanrıça olduğunu düşünebiliriz.

\section{b.KUB 7.36 (Bo 4912) (Yeni Hitit) $)^{25}$}

ay. sağ.kr. $3^{\prime}, 1^{\prime}$

$\mathbf{x}+\mathbf{1}[n a-a \check{s}-t a]$ ha-an-te-ez-zi pal-ši A-NA [(T.A.)]

2' [ši-p] a-an-ti EGIR-an-da-ma-k[án 1 MÁŠ.GAL $A$ - $N A$ [(T.A.)]

3' 1 MÁŠ́.GAL-ma-kán $A$-NA ${ }^{\mathrm{D}} K a p-\left[p a-r i-i a-m u{ }^{\mathrm{D} L A M M A}{ }^{\mathrm{URU}} T a-t u-\check{s} u-n a\right]$

$\mathbf{4}^{\prime}$ DAMMA URU $T a-a s ̌-h a-p u-n a[\grave{U} A-N A$ (T.A.)]

$\mathbf{5}^{\prime}$ ši-pa-an-ti na-aš ǐ̌-ta-n[a-ni pi-ra-an da-a-i]

22 Diğer anlamları için bibliyografya ile birlikte bk. Nursel Aslantürk, Hitit Belgelerinde Hurri Tannlan ve Onlarn Kültteri, Ankara Üniversitesi Sosyal Bilimler Enstitüsü, Eskiçağ Dilleri ve Kültürleri (Hititoloji) Anabilim Dalı, Yayımlanmamış Doktora Tezi, Ankara, 2013, s. 25-26.

23 McMahon, age., s. 18; Steiler, age., s. 291dn.909.

24 Hans Gustav Güterbock, "Hittite Religion", Forgetten Religions, 1950, s. 92; McMahon, age., s. 26; Alfonso Archi, "How a God of Nature Became a Tutelary God of The King", IX. Uluslararast Hititoloji Kongresi Bildirirleri, 1.Cilt, 2019, s. 54.

25 McMahon, age., s. 168-171; Ayrica bk. Haas, age., s. 599 dn.410. 


\section{Tercüme:}

$\mathbf{x}+\mathbf{1}$ [Ve sonra] ilk kez [(Tanrı/ça x)] için

$\mathbf{2}^{\prime}$ [kur]ban sunar. Sonradan [ise bir keçi [(Tanrı/ça x)] için

3' Bir keçi ise Tanrıça Kap[pariiamu'ya, Tatušna Kenti Koruyucu Tanrısına]

4' Tašḩapuna Kenti Koruyucu Tanrısına [ve Tanrı/ça x'e]

$\mathbf{5}^{\prime}$ kurban sunar. Ve onları suna[ğa, (sunağın) önüne(?) koyar.]

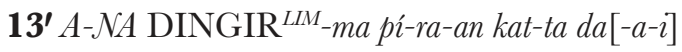

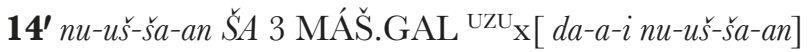

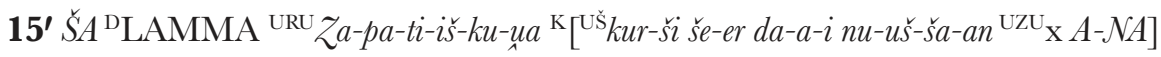

$\mathbf{1 6}^{\mathrm{D}}$ Kap-pa-ri-ia-mu ${ }^{\mathrm{D} L A M M A}{ }^{\mathrm{URU}} T[a-t u-s ̌ u-n a]$

$17{ }^{\circ} \mathrm{LAMMA}{ }^{\mathrm{URU}} \mathrm{Ta}-a \check{s}^{-} h a-p u-n a \dot{U}$ [A-NA (T.A.)]

$\mathbf{1 8}^{\prime}$ Kuškur-ši še-er da-a-i[

$\mathbf{1 9}^{\prime}[\quad] \times \times \times x$

\section{Tercüme:}

13' Tanrının önüne de koyar.

$\mathbf{1 4}^{\prime} \mathrm{O}$, üç keçinin x e[tini düzenler. Ve onları]

15' Zapatiškuuua Kenti Koruyucu Tanrısı'nın [kurša'sına koyar. x etini]

16' Tanrıça Kappariijamu, T[atušuna Kenti Koruyucu Tanrısı]

17' Tašhapuna Kenti Koruyucu Tanrısı ve [Tanrı/ça x’in]

$1 \mathbf{1 8}^{\prime}$ kur'̌́a'sina koyar[.

$\mathbf{1 9}^{\prime}[\quad] \times \times \times \times[$

\section{Metnin Değerlendirmesi:}

Metinde bir keçinin Tanrıça Kappariiamu’ya, Tatušna Kenti Koruyucu Tanrısına ve Tašḩapuna Kenti Koruyucu Tanrssına kurban olarak sunulduğu anlatılmaktadır. Adının Koruyucu Tanrının kurša-'sı ile bağlantılı geçtiği bu metnin 
15-18. satırlarında ise, x etini Tanrıça Kappariiiamu, Tatušuna Kenti Koruyucu Tanrısı, Tašhnapuna Kenti Koruyucu Tanrısı ve ne yazık ki adını okuyamadığımız bir tanrı için kuř̌a-ya koydukları anlatılmaktadır.

Tanrıçanın adı, Koruyucu Tanrı için yapılan festivale ait fragmanda (CTH 685) geçmektedir:

\section{a.KUB 53.11(Bo 2309) $\left(\text { Orta Hitit }^{26}\right)^{27}$ II $9^{\prime}$, 18'$^{\prime}, 2^{\prime}$, 31' $^{\prime}$ öy.II}

9' 1 NINDA $L A-A B-K U-m a$ pár-šsi-ia na-an-ša-an ${ }^{\mathrm{D}} K a p-p[a-r i-i a-m u-i]^{28}$

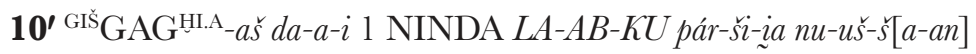

$\mathbf{1 6}^{\prime}$ na-aš ap-ap-pa ti-i-e-ez-zi nu-kán Giš ${ }_{e-e r-h u-i a-a z}$

17 me-ma-al ha-aš-šu-un-ga-a-iz-zi nu-uš-ša-an iš-ta-na-a-ni

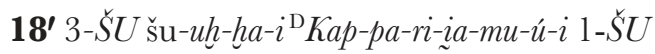

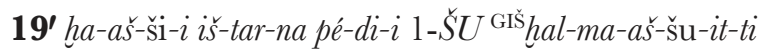

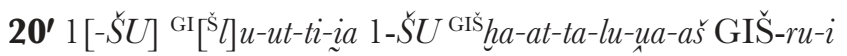

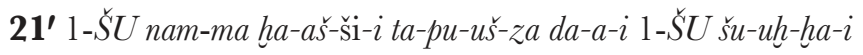

24' 1 UZU NÍG.GIG-ma 1 UZUŠ S̀ ar-ḩa kur!-aš-ki-i[z-z]i

25' nu-uš-ša-an ${ }^{\mathrm{D}} K a p-p a-r i-i a-m u-i d a-a-i h\left[a-a \check{S}^{-}-5\right] i-i$

26' iš-tar-na pé-e-di da-a-i GIŠ hal-ma-aš-šu-it-ti

$27^{\prime}$ GIšha-at-ta-lu-ua-aš GIŠ-ru-i nam-ma ha-aš-ši- $i$

$\mathbf{2 8}^{\prime} t a-p u-u \breve{s}^{\prime} z a$ da- $a-i$

$2 \mathbf{2 9}^{\prime} n u-z a{ }^{\mathrm{LU}} \mathrm{SANGA}{ }^{\mathrm{D} L A M M A}{ }^{\mathrm{URU}} H A-A T-T I 1{ }^{\mathrm{DUG}} K U-K U-U B$ ta- $a-u-u[a-l a-a s]$

26 McMahon, age., 1991, s. 236'da "Eski Hitit" olarak değerlendirmiştir.

27 McMahon, age., 1991, s. 236, 241; Erich Neu, Ein althethitisches Gereitterritual, StBoT 12, Wiesbaden. 1970, s. 71; ayrca bk. Heinrich Otten, Ein hethitisches Festritual (KBo XIX 128), StBoT 13, Wiesbaden 1971, s. 32; Yoshida, age., s. 213-214; Andrej V. Sideltsev, "An Overlooked Case of Inversion in Middle Hittite?", Fs Košak, 2007, s. 616.

28 Krş. Neu, StBoT 12, s. 71 dn. 15. 
30' da-a-i nu iš-ta-na-a-ni pi-ra-an 3- $\breve{S} U$ ši[-pa-an-ti]

$31^{\prime}{ }^{\mathrm{D}} K a p-p a-r i-i a-m u$ 1-Š́ $U$ ha-aš-ši-i iš-tar-na pé [-e-di]

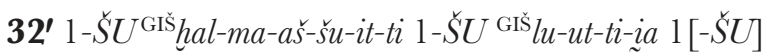

\section{Tercüme:}

9 Bir nemli ekmeği ise parçalar. Ve onu Tanrıça Kapp[ariiamu] için

10 kazıklara (çivilere) koyar. Bir nemli ekmeği ise parçalar. Ve onu ocağın ortasına (orta yerine) koyar.

$\mathbf{1 6}^{\prime}$ Ve geri gider. Sepet ile

17' iri öğütülmüş unu serper. Ve onu sunağa

$\mathbf{1 8}$ ' üç kez döker. Bir kez Tanrıça Kapp[ariiamu] için

19 bir kez ocağın ortasına (orta yeri için), bir kez taht için,

$\mathbf{2 0}^{\prime}$ bir [kez] pencere için, bir kez kapının sürgüsü için,

$\mathbf{2 1}$ ' bir kez ayrıca ocağın yanına döker.

24' Bir karaciğeri ve de bir kalbi kese[r].

25' Onları Tanrıça Kappariiiamu için koyar. O[cağı]n

$\mathbf{2 6}^{\prime}$ ortasına (orta yeri için) koyar. Tahta (taht için) [

27 kapının sürgüsü için, ayrıca ocağın[

$\mathbf{2 8}$ ' yanına koyar. [

29' Ve Hatti Kentinin Koruyucu tanrısının rahibi bir (ibrik) kap tau [al] içkisini

$\mathbf{3 0}$ ' alır. Ve üç kez sunağa lib[asyon yapar].

31' Bir kez Tanrıça Kappariiamu'ya, bir kez ocağın ortasına (orta yerine)

32' bir kez tahta, bir kez pencereye 


\section{Metin Değerlendirmesi}

Festivale ait bu metni bir bütün olarak ele aldığımızda (öy.I ve öy.II) farklı türde kurban sunumlarının anlatıldığını görmekteyiz:

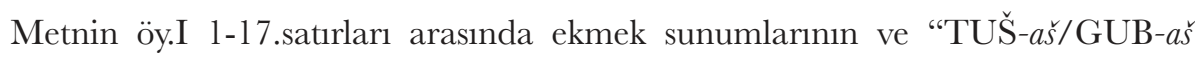
eku- "oturarak içmek, ayakta içmek" seremonilerinin yapıldığı anlatılmaktadır. Ne yazık ki öy.I'in oldukça tahrip olmasından dolayı, söz konusu sunumların nasıl yapıldığını tam olarak öğrenemesek de Hatti Kentinin Koruyucu Tanrısına ve Maraššanta nehrine yapıldığı anlatılmaktadır.

Öy.II'de ise 1-6 ve 22-28.satırlar arasında karaciğer, kalp, baş vb. gibi çeşitli et, 7-21'de ekmek ve un, 29-32.satırlarda ise Hatti Kentinin Koruyucu tanrısının rahibi tarafindan $K U K U B U$ kapında taual içkisinin sunulması anlatılmaktadır. Bu farklı türde sunumların ise ocak, taht pencere, kapının sürgüsü vb. gibi tapınağın kutsal bölümlerine/yerlerine ve Tanrıça Kappariinamu'ya sunulduğu metinde belirtilmektedir. Kutsal yerlerin sıralandığı bu metinde, yalnızca tanrıçanın adının geçmesi ise oldukça ilgi çekicidir. Burada hemen belirtmek gerekirse, bu metin Tanrıça Kappariianamu'nun, şimdiye kadar neşredilmiş olan tapınakların kutsal yerleri listesinde adının geçtiği tek belgedir. Metnin öy.II 9-10'da Tanrıçanın adaklarınıçivilerin/kazıklarınüzerinde almasından sözedilmektedir. Bu kazıkların/ çivilerin, KUB 55.43 I 10'daki kazıklara benzerlik gösterdiği düşünülmekte; ancak bunların Tanrıça ile olan bağlantısı belirtilmemektedir. Söz konusu kazıkların/

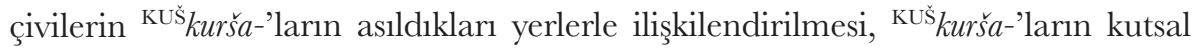
mekanlar/yerler listesinde sıklıkla karşımıza çıkması ve Tanrıça Kappriiąamu'nun adının ise yalnızca kutsal yerlerin sıralandığı bu listede geçmesini göz önünde

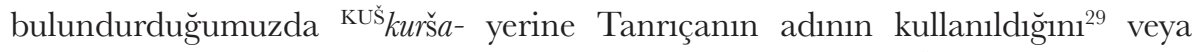

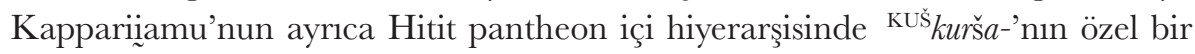
sembolü olduğunu, tıpkı tanrı/ça atribüleri gibi ${ }^{30}$, gösterebilir.

29 Ayrica bk. Maciej Popko, Kultobjekte in der hethitischen Religion (nach keilschriftlichen Quellen), Warszawa, 1978, s. 112 ve dn.77.

30 Tanrı ve tanrıça atribüleri için bk. Aslantürk, Yayımlanmamış Dokora tezi, 2013. 


\section{b.KUB 55.43(Bo 2393+Bo 5138)+DBH 43/2.4(Bo 8272)+KBo 69.172(E 1491) (Orta Hitit $)^{31}$}

\section{öy.II}

13 EGIR-an-da-ma DLAMMA URU Za-pa-ti-iš-ku-ua GUB-aš $a-k u-u a-a n[-z i]$

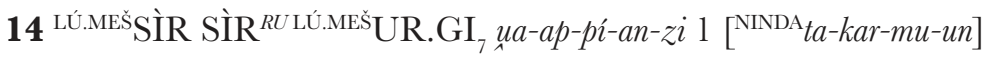

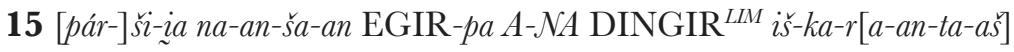

$16[\check{s}$ e-e $] r$ ti-an-zi EGIR-an-da-ma Ki-pi-ik-ki-iš-d[u]

$17\left[{ }^{\mathrm{D}} K a\right] p-p a-r i-\underset{\sim}{i} a-m u-u n{ }^{\mathrm{D}} \mathrm{LAMMA}{ }^{\mathrm{URU}} \mathrm{Ta}_{a}-t a-\check{s}^{2} u-n a[\mathrm{]}$

$18{ }^{\mathrm{D}} \mathrm{LAMMA}{ }^{\mathrm{URU}} T a-a s ̌-h a-p u-n a$ GUB-aš $a-k u-u a-a n-z i 3^{\mathrm{NINDA}}[t a-k a r-m u-u s ́]$

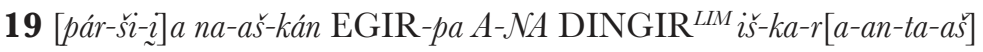

20 [še-er ti-an-z]i LÚ.MEŠSİR SİR ${ }^{R U}$ LÚ.MEŠUR.GI $_{7} u a-a[p-p i-a n-z i]$

\section{Tercüme:}

13 Sonradan ise Zapatiškuụa Kenti Koruyucu Tanrısı (için) ayakta içerl[er.]

14 Şarkıcılar şarkı söylerler. Köpek adamlar havlarlar. Bir [takarmu ekmeğini]

15 [par]çalar. Ve onu tekrar tanrı için sıralananların,

16 [üz] erine koyarlar. Sonradan ise Kipikkišd $[\mathrm{u}]$

17 [Tanrıça Ka]pparịiamu, Tatašuna Kenti Koruyucu Tanrısı [ ]

18 (ve) Tašhapuna Kenti Koruyucu Tanrısı (için) ayakta içerler. Üç (adet) [takarmu] ekmeğini

19 [parçal] ar. Ve onları tekrar tanrı için sıralana[nların]

20 [üzerine koyarla]r. Şarkıcılar şarkı söylerler. Köpek adamlar hav[larlar.]

\section{IV}

$\mathbf{1 6}^{\prime} \ldots n{ }^{\mathrm{D}} K a p-p a-r i-i \underset{\sim}{-} a[-m] u$

$17{ }^{\mathrm{D}} \mathrm{LAMMA}{ }^{\mathrm{URU}} T a-t a-\mathrm{s} u-n a{ }^{\mathrm{D}} \mathrm{LAMMA}{ }^{\mathrm{URU}} T a-a s ̌-h a-p u-n a \mathrm{GUB}-a \check{s} a-k u-u a-a n-z i$

18' $2{ }^{\mathrm{NINDA}} t a-k a r-m u-u s ̌$ pár-ši-ia na-aš-kán EGIR-pa ${ }^{\mathrm{KUŠ}} k u r-s ̌ i d a-a-i[$

19 $^{\prime}$ LÚ.MEŠSİR SÌR ${ }^{R U ~ L U ́ . M E S ̌ U R . G I ~}{ }_{7} a a-a p-p i-a n-z i$

31 McMahon, age., s. 148, 149, 154, 155; Groddek, 2002a: 75, 77, 78; ayrica bk. Haas, age., s. 267 dn.113; 455 dn.51; 458 dn.68, 74, 78; 618 dn.6; Yoshida, age., s. 219-220; Taggar-Cohen, age., s. 303; Steiler, age., s. 290. 


\section{Tercüme:}

16' $^{\prime}$... Ve Tanrıça Kappariija $[\mathrm{m}] \mathrm{u}$,

17' Tatašuna Kenti Koruyucu Tanrısı (ve) Tašḩapuna Kenti Koruyucu Tanrısı (için) ayakta içerler.

18' İki (adet) [takarmu] ekmeğini [parçal]ar. Ve onları tekrar kurša'ya koyar. [

19 'Şarkıcılar şarkı söylerler. Köpek adamlar hav[larlar.]

\section{Metin Değerlendirmesi}

Metinden Tanrıça Kappariỉamu, Kipikkišdu, Tatašuna Kenti Koruyucu Tanrısı ve Tašḩapuna Kenti Koruyucu Tanrısı için ayakta içildiği adı geçen tanrı(ça) lara üç adet takarmu ekmeğinin parçalandığı, parçalanan ekmeklerin tanrılar için sunulanların üzerine konulduğu seremoniye şarkıcıların şarkı söyleyip köpek adamların (köpek maskesi takmış) havlamalarıyla devam edildiği öğrenilmektedir.

\section{öy.II, IV}

str.14, 19': uappiianzi: uappiie/a-; uapiia- "havlamak" Prs. 3.Ç.Ş. haldedir.

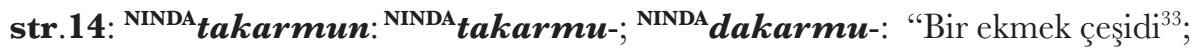
kek $^{34}$; pasta ${ }^{35}$;" anlamina gelen kelime Sg.Akk.com. haldedir. Hoffner, Aliementa, 1974, s.185'te bu ekmek çeşidinin adının Hattice olabileceğini ve ekmeğin adıyla Takarmuha kentinin (URU Takarmuha: KBo 10.11 öy.I 3) adı arasında bir bağlantı olduğunu ifade etmektedir. Ancak bu kentin adını, H.Ertem, Boğazköy metinlerinde geçen coğrafya adları dizini, 1973, s.131'de "Takir'muha: KBo 10.11 öy.I 3 " G.F. del Monte RGTG 6, 1978, s.383'te ise "URU Ta-kal-mu-ha: KBo 10.11 öy.I 3" olarak vermektedir. "Takarmuha” kentinin adının geçtiği metin yerini incelendiğimizde ise "kar" işaretinin oldukça tahrip olduğunu görmekteyiz:

32 HW: 244; EDHIL: 958; MHH: 785.

33 HW: 204; Harry A. Hoffner, Alimenta Hethaeorum. Food Production in Hittite Asia Minor, New Haven 1974, s. 185; HEG (t,d/1): 37; HHw: 160.

34 МHH: 668.

35 Ünal, age., s. 502. 


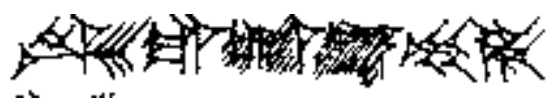

Kentin adının farklı farklı okunması, G. F. del Monte-J.Tischler, RGTC 6, 1978; P.Cornil, Liste des noms géographiques des textes hittites. KBo XXIII-XXX, XXXIII, KUB XLV-LVII", 1990; G. F. del Monte, RGTG 6/2, 1992 ve 1992 sonrası yayınlanmış Boğazköy'de gün yüzüne çıkartılmış Hitit çiviyazılı tabletlerde de geçmemesinden dolayı kentin adının "Takalmuha" olarak okunması gerektiğini düşünmekteyiz ${ }^{36}$.

iškarantaš: iškar-; išgar-; išqar-; "sokmak, delmek, kesmek, batırmak, saplamak, dikmek, dizmek, şişe dizmek, sıralamak, süslemek; (dansta) dizilmek, (ekmekte) siralamak, siraya girmek, çember oluşturmak"37.

iškarant- Ptzp.; iškarantaš: Pl. Gen.

c.KBo 21.89(402/d)(+)KBo 8.97(230/m) Orta Hitit dönemine tarihlendirilen metin ise ne yazık ki içerik açısından çok fazla bilgi vermemektedir:

öy. $\mathbf{I}^{38}$

16' $\left[n u A-\mathcal{N} A{ }^{\mathrm{D} U T U}{ }^{\mathrm{D} I M}{ }^{\mathrm{D}} \mathrm{LAMM}\right] \mathrm{A}{ }^{\mathrm{URU}} H A-A T-T{ }^{\mathrm{D}} K a p-p a-r i-\underset{\sim}{-} a-m u-u ́-i$

$17 '$ [ $\left.{ }^{\mathrm{D}} \mathrm{LAMM}\left(\mathrm{A}{ }^{\mathrm{URU}} T a-a s ̌-h a-p u-n\right) a\right]{ }^{\mathrm{D}} \mathrm{LAMMA}{ }^{\mathrm{URU}} T a-a-t a-\check{\mathrm{s} u}-n a$

18' [ $\left.{ }^{\mathrm{D}}\left(\mathrm{LAMMA}{ }^{\mathrm{URU}} H a-t i-e n-z u\right)\right]-u a{ }^{\mathrm{D}} H a-a s ̌-g a-l a-a-i$ DL[AMMA URU $] Z a-p a-t[i-i]$ š- $k u-u u_{1}$

"Firtına Tanrısının Adamı" olarak adlandırılan festival fragmanı (CTH 652) olan ve Yeni Hitit dönemine tarihlendirilen KBo 52.128(1332/u)(+) KUB 55.54 (Bo 2381(+)Bo 3470)+Bo 7825(+)DBH 43/2.13(Bo 8281)(+) VSNF 12.55 (VAT 13589) metinden Tanrıça Kapparïiamuu iça için bir kadeh ve bir kalın ekmeğin hazırlanıldığı öğrenilmektedir:

36 Ayrica bk. HEG (t,d/ 1): 37

37 HW: 88, HHw: 65, HEG: 399: "stechen; hineinstecken, feststecken"; HED (a,e,i): 416-419 "sting, prick, stab, pierce, skever, stick, affix, fasten,attach, set, post; (instr.) stew, heck, cleave, cling (to)" ; EDHIL: 401: "to sting, to stab, to pierce, to stick(to)"; ayrıca filin kökünü HED (a,e,i): 416-419'da "iškarra-; iškarriia-"; MHH:273'te ise "iškanniia-; iškarannai-" da olarak verilmektedir.

38 McMahon, age., s. 175; ayrica bk. Hans Martin Kümmel, Ersatzrituale für den hethitischen König, StBoT 3, Wiesbaden 1967, s. 88; Sideltsev, agm., s. 616. 


\section{öy..$^{39}$}

$\mathbf{2 9}^{\prime}[A-\mathcal{N}] A^{\mathrm{D}} \mathrm{U}^{\mathrm{URU}} \mathcal{N}_{e}-r i-i k 1$ GAL 1 NINDA.KUR ${ }_{4}$.RA $A-N A{ }^{\mathrm{D}} Z A-B A_{4}-B A_{4} 1 \mathrm{G}[\mathrm{AL}$ 1 NINDA.KUR 4 .RA]

$\mathbf{3 0}^{\prime}[A-N] A{ }^{\mathrm{D}} K a p-p a-r i-i a-\mathrm{A} . \mathrm{A} 1$ GAL 1 NINDA.KUR ${ }_{4}$.RA $A$-NA ${ }^{\mathrm{D} L A M M A}$ GIŠ ${ }^{D}$ INANN[A

31' $A$ - $N A{ }^{\text {DLAMMA }}{ }^{\text {GIŠŠUKUR } 1 \text { GAL } 1 \text { NINDA.KUR }}$.RA ...

\section{Tercüme:}

29' Nerik Kenti Fırtına Tanrısı için bir kadeh, bir kalın ekmek, Savaş Tanrısı için bir k[adeh (ve) bir kalın ekmek]

30' Kappariiamu(uxa) için bir kadeh (ve) bir kalın ekmek, Koruyucu tanrı(nın) 1[ir için bir kadeh (ve) bir kalın ekmek]

31' Koruyucu tanrının mızrağı için bir kadeh, bir kalın ekmek...

Yeni Hitit dönemine tarihlendirilen ve Büyük festival fragmanı olan (CTH 669) metinlerde de tanrıçanın adı geçmektedir:

\section{KBo $11.35(326 / \mathbf{p})^{40}$}

ay.

$1{ }^{\mathrm{D}} \mathrm{U}^{\mathrm{D}} \mathrm{U}$ URU Zi[-ip-pa-la-an-da

$2{ }^{\mathrm{D}} \mathrm{LAMMA}{ }^{\mathrm{D}} \mathrm{Ha}-p a-a n-t[a-l i-i a$

$3{ }^{\mathrm{D}} K a p-p a-r i-i a-m u-u[n$

$4{ }^{\mathrm{D}} \mathrm{Te}-\mathrm{li}-p i-n u-u n{ }^{\mathrm{D}}$ [Ha-te-pi-nu

$5{ }^{\mathrm{D}} \mathrm{Pu}-u \check{s}^{\leftarrow}-k u-r u-n a-a$

$6{ }^{\mathrm{D}} U^{-r i-i a-d u}$

39 Transk. ve terc. tarafimızdan yapılmıştır; ayrıca bk. Groddek, 2002a: 97; Detlev Groddek, Albertine Hagenbuchner, Inge Hoffmann, Hethitische Texte in Transkription. VS NF 12, DBH 6, Dresden 2002, s. 92; René Lebrun, "Reflexions Concernant Hapaliya et la cite de sura". ArAn 4: 2000, s. 114-115.

40 Transk. ve terc. tarafimızdan yapılmıştır; ayrıca bk. Haas, age., s. 487 dn. 168. 
1 Firtına Tanrısı, Zi[ppalanda Kenti Firtına Tanrısı

2 Koruyucu Tanrı, Tanrıça Ḩapant[aliia

3 Tanrıça Kappariiamu[

4 Tanrı Telipinu, Tanrı[ça Hatepinu

5 Tanrı Puškuruna

6 Tanrı Uriiadu

KUB 20.39 (Bo 3247 $)^{41}$ metinden ise tanrıçanın dokuz tanrı ile birlikte, kral tarafından içmek eylemiyle kutsandığını öğrenmekteyiz. Söz konusu metni; KBo 11.35(326/p)'e göre tekrarlanarak geçen benzer kült işlemleri yardımıyla da şu şekilde rekonstüre edebiliriz:

\section{ay. V}

11' LUGAL-uš GUB- $a s ̌ 5[e-k u-z i]$

$12^{\prime}{ }^{\mathrm{D}} \mathrm{U}{ }^{\mathrm{U}} \mathrm{U}$ URU $z i[-i p-p a-l a-a n-d a$

$\mathbf{1 3}^{\mathrm{D}} I-n a-a r{ }^{\mathrm{D}} H[a-p a-a n-t a-l i-i a$

$\mathbf{1 4}^{\mathrm{D}}$ Kap-pa-ri-ia-m[u-un

$\mathbf{1 5}^{\mathrm{D}}{ }^{\mathrm{D}} T e-l i-p i-n u-u n{ }^{\mathrm{D}} H\left[a-t e-p i-n u \quad . . . . .{ }^{\mathrm{D}} P u-u s^{2}-k u-r u-n a-a{ }^{\mathrm{D}} U^{\prime}-r i-i a-d u\right.$

11' Kral ayakta dokuz kez [içer]:

12' Firtına tanrısı, Zi[ppalanda Kenti Firtına Tanrısı,

13' Tanrı Inar, Tanrıça Hु[apantaliya,

$\mathbf{1 4}^{\prime}$ Tanrıça Kappariijam[u,

15' Tanrı Telipinu, Tanrıça Hู[atepinu ...... Tanrı Puškuruna, Tanrı Uriiadu

Kralın dokuz tanrı için içtiği bu metinde; kutsama eyleminin kral tarafından gerçekleştirildiği öğrenilmektedir. Tanrıça Kappariịamu'nun adının, Hapantaliia’dan sonra geçmesi ise tanrıçanın, Ḩapantaliia'dan sonra kutsandığını göstermektedir. 


\section{Sonuç}

Hititler'in başkenti Boğazköy başta olmak üzere diğer Hitit yerleşim yerlerinde de yapılan arkeolojik kazılarda gün yüzüne çıkartılan Hitit çiviyazılı tabletlerin büyük bir bölümünün dini içerikli olması Hititler'in teokratik-tek erklik nitelikli bir yönetim biçimine sahip olduklarını göstermektedir. Hititler kuruluşlarını tamamlayıp dış politikada istilacı bir strateji benimsediklerinde; yani siyasi ve askeri olarak geliş̧ikleri dönemde, ele geçirdikleri ülkelerin tanrılarını kendi tanrılar topluluğuna katmışlardır. Hititler benimsedikleri bu yayılım politikasıyla; zaptettikleri ülkelerin dinsel ve kültürel hasasiyetlerini merkeze taşımışlardır. Böylece siyasal kültürlerini çeşitlendirmişlerdir. Bunun sonucu olarak ele geçirdikleri ülkelerin tanrılarını kendi pantheonlarına taşıyarak; pantheonlarındaki tanrı ve tanrıçalarının sayılarını artırmışlardır. "Bin tanrılı toplum" olarak tanımladığımız Hititler'e ait çiviyazılı metinlerde geçen tanrı ve tanrıça adlarının çeşitliliği de bunu kanıtlamaktadır.

Hititolojinin gözde konularından olan, Hitit pantheon araştırmalarında, kutsal varlıkların cinsel kimliklerinin belirlenmesi oldukça güçtür. Hitit çiviyazılı metinlerde kutsal varlıklar "Tanrı" anlamma gelen "DINGIR" determinativiyle belirtilmektedir. Ancak bu determinativ metinlerde kutsal varlığın eril ya da dişil tanımlamasını tam olarak ortaya koymamaktadır. Bu nedenle de Hitit pantheonunda yer alan tanrı ve tanrıçaların; plastik yapıtlarda (yontu, rölyef ve figürin) tasvir edilmediği ve çiviyazılı metinlerde çift olarak tanımlanmadı̆̆ı sürece cinsel kimliklerinin tanımlanması oldukça zordur. Bu bağlamda Hitit tanrılar topluluğunun bir üyesi olan Hatti kökenli Kappariiamu'nun bugüne kadar yapılan bilimsel çalışmalarda cinsel kimliğinin belirlenmesi konusunda da farklı görüşlerin mevcut olduğu görülmektedir. "Kappariiiamuựa" adının analizinden yola çıarak "güç kuvvet ve kudret anlamına gelen "muи a-" kelimesi daha çok kutsal varlığın eril olduğunu düşündürtse de Hititçe kadın şahıs adlarında da "muuxa-" kelimesinin bulunmasi; Hepamuư⿱ (NH 364), Muựanani (NH 835), Muuxatti (NH 838); ve "LAMMA" ideogramıla yazılan tüm Koruyucu ilahların erkek yani tanrı olarak tanımlanılmasından yola çıkarak çalışmamızda adı geçenin cinsel kimliğini dişil yani "tanrıça" olarak kabul edebiliriz.

Bu bağlamda çalışmamızda Hitit tanrılar topluluğunun bir üyesi olan Tanrıça Kappariiamu'nun adının ilk kez Eski Hitit dönemine tarihlendirilen metinlerde geçmesi tanrıçanın Eski Hitit Döneminden itibaren Hitit pantheonunda kutsandığını göstermektedir. Hitit teopolitiğine göre; genellikle festival metinlerinde

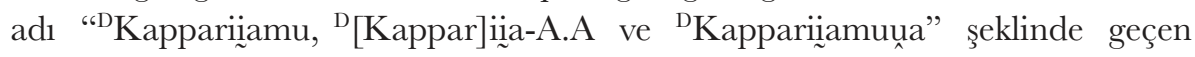


tanrıçanın KI.LAM bayramında kral tarafindan kutsandığı ve adının Tahantitu ile birlikte geçtiği görülmektedir.

Çalışmamızda temelde vurgulanması gereken bir başka nokta Tanrıçanın adının geçtiği Hitit çiviyazılı metinlerin tümünün bir araya getirilmesiyle, metinlerin transkripsiyon, tercüme ve dil bilimsel incelemelerinden elde edilen veriler doğrultusunda KUB 20.39(Bo 3247) numaralı metin; KBo 11.35(326/p)'e göre söz konusu metinde tekrarlanarak geçen benzer yapımlı kelimeler ve kült işlemleri yardımı ile rekonstüre edilebilinmiştir.

Tanrıçanın; Koruyucu Tanrının av çantasının (kurša-) yenilenmesine ait metinlerde, Koruyucu Tanrı için yapılan festivale ait metinde geçmesi ve genellikle koruyucu tanrlarla birlikte (Tatašuna Kenti Koruyucu Tanrısı ve Hatenzuựa Kenti Koruyucu Tanrıs1) kutsanmasindan dolay1; Kappariiamu'nun koruyucu bir tanrıça olabileceği ve bu tanrılarla bir üçlü oluşturduğu sonucuna ulaşabiliriz.

Tanrıça Kappariinamu'nun, şimdiye kadar neşredilmiş olan tapınakların kutsal yerleri listesinde adının geçtiği tek belge olan KUB 53.11(Bo 2309) metinde yalnızca tanrıçanın adının geçmesi Kư̌šr kurša ile tanrıça arasında bir ilgi olabileceğini göstermektedir. Ancak bu ilgiyi ortaya koyabilmek için ne yazık ki elimizde yeteri kadar belge bulunmamaktadır. Şüphesiz ki bundan sonraki yıllarda toprak altından çıkan Hitit çiviyazılı tabletler üzerinde yapılan bilimsel çalışmalardan elde edilecek veriler Kư̌kurša ile tanrıça arasındaki ilgiyi daha iyi aydınlatacaktır. Söz konusu metin; “Kuškuř̌a-” yerine Tanrıça Kappriiiamu'nun adının kullanılmış

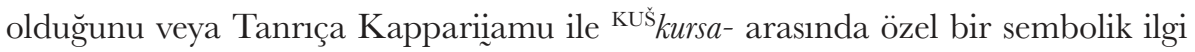
olabileceğini ima etmektedir.

Tüm bu veriler adı geçen Tanrıçanın Hitit pantheonunda teolojik sınıflamada ya da pantehon içi hiyerarşide ikincil pozisyonlarda konumlanan bir tanrıça olduğunu düşündürtmektedir. 


\section{KAYNAKLAR}

Akdoğan, Rukiye, Hethitische Texte Bo 4658-Bo 5000. Teil 1: Transkriptionen. Teil 2: Autographien, DBH 46, Wiesbaden 2016.

Alp, Sedat, Beiträge zur Exforschung des hethitischen Tempels. Kultanlagen im Lichte der Keilschrifttexte. Neue Deutungen, Ankara 1983.

Archi, Alfonso, "How a God of Nature Became a Tutelary God of The King", IX. Uluslararasi Hititoloji Kongresi Bildirileri, 1. Cilt, (2019), s. 49-63.

Arıkan, Yasemin, "Hitit Dini Üzerine Bir İnceleme”, AÜDTCF Dergisi, C 38/S. 1-2, (1996). s. 271-285.

Arıkan, Yasemin, "Hitit Dininin Tarihi Gelişimi ve Hitit Panteonu”, Yaşar Coşkun’a Saygr Yazıları, 2018, s. 41-72.

Aslantürk, Nursel, Hitit Belgelerinde Hurri Tannlan ve Onlarn Kültleri, Ankara Üniversitesi Sosyal Bilimler Enstitüsü, Eskiçağ Dilleri ve Kültürleri (Hititoloji) Anabilim Dalı, Yayımlanmamış Doktora Tezi, Ankara 2013, s. 25-26.

Badalì, Enrico, Strumenti musicali, musici e musica nella celebrazione delle feste ittite, THeth 14/1, Heidelberg 1991.

Bawanypeck, Daliah, Die Rituale der Auguren, THeth 25, Heidelberg 2005.

Cornil, Pierre. "Liste des noms géographiques des textes hittites. KBo XXIIIXXX, XXXIII, KUB XLV-LVII", Hethitica 10, (1990) s. 7-108.

del Monte Giuseppe F., Die Orts- und Gewässernamen der hethitischen Texte. Supplement, RGTC 6/2 Wiesbaden 1992.

Doğan-Alparslan, Meltem Hitit krah II. Muwatalli: Kişsiliği ve İcraatı (Filolojïk Belgeler Işı̆̆gnda), Ege Yayınları, İstanbul 2018

Ertem, Hayri, Boğazköy Metinlerinde Geçen Coğrafya Adlan Dizini, Ankara 1973.

Groddek, Detlev, Hethitische Texte in Transkription. KBo 30, DBH 2, Dresden 2002.

Groddek, Detlev, Hethitische Texte in Transkription. KUB 55, DBH 4, Dresden 2002.

Groddek, Detlev, Hagenbuchner, Albertine, Hoffmann Inge, Hethitische Texte in Transkription. VS NF 12, DBH 6, Dresden 2002.

Groddek, Detlev, Hethitische Texte in Transkription. KUB 59, DBH 14, Dresden 2004. 
Groddek, Detlev, Hethitische Texte in Transkription. KUB 20, DBH 13, Dresden 2004.

Groddek, Detlev, Hethitische Texte in Transkription. KUB 60, DBH 20, Wiesbaden 2006.

Güterbock Hans, Gustav, Hittite Religion, Forgetten Religions, (1950) s. 81-109.

Frantz-Szabó, Gabriella, Kappariiamu, RlA 5, (1976-1980), s. 400.

van Gessel, Ben H.L. Onomasticon of the Hittite Pantheon, Part I, Leiden-NewyorkKöln 1998.

Haas, Volkert, Geschichte der Hethitischen Religion, HbOr I/15, Leiden 1994.

Hoffner, Harry A., Alimenta Hethaeorum. Food Production in Hittite Asia Minor, New Haven 1974.

Pecchioli Daddi, di Franca, Osservazioni su alcuni teonimi hattici. Fs Alp, (1992) s. 97-108.

Klinger, Jörg, Untersuchungen zur Rekonstruktion des hattischen Kultsichicht, StBoT 37, Wiesbaden 1996.

Kümmel, Hans Martin, Ersatzrituale für den hethitischen König, StBoT 3, Wiesbaden 1967.

Laroche, Emmanuel, Les noms des Hittites, Paris 1966.

Laroche, Emmanuel, Recherches sur les noms des dieux hittites, (RHA VII/46) Paris 1946-1947.

Lebrun, René, Reflexions Concernant Huapaliya et la cite de surá. ArAn 4, (2000), s. 113-120.

Monte Giuseppe F. del-Tischler Johann, Die Orts- und Gewässernamen der hethitischen Texte, RGTG VI, Wiesbaden 1978.

McMahon, Gregory, The Hittite State Cult of the Tutelary Deities, AS 25, Chicago 1991.

Neu, Erich, Ein althethitisches Gewitterritual, StBoT 12, Wiesbaden 1970.

Neu, Erich, Althethitische Ritualtexte in Umschrift, StBoT 25, Wiesbaden 1980.

Otten, Heinrich, Ein hethitisches Festritual (KBo XIX 128), StBoT 13, Wiesbaden 1971. 
Otten, Heinrich, Kapperi, RlA 5, (1976-1980), s. 400.

Popko, Maciej, Kultobjekte in der hethitischen Religion (nach keilschriftlichen Quellen), Warszawa 1978.

Schuler, Einar von, Die Kašäer. Ein Beitrag zur Ethnographie des alten Kleinasien, Berlin 1965.

Sideltsev, Andrej V., "An Overlooked Case of Inversion in Middle Hittite?", Fs Košak, (2007), s. 613-629.

Singer, Itamar, The Hittite KI.LAM Festival, StBoT 27, Part: I, Wiesbaden 1983.

Singer, Itamar, The Hittite KI.LAM Festival, StBoT 28, Part: II, Wiesbaden 1984.

Soysal, Oğuz, Hattischer Wortschatz in hethitischer Textüberlieferung, HbOr I/74, Leiden 2004.

Starke, Frank, Untersuchungen zur Stammbuilding des kelischrift-luwischen Nomens, StBoT 31, Wiesbaden 1990.

Steiler, Charles W., The Solar Deities of Bronze Age Anatolia, Studies in Texts of the Early Hittite Kingdom, StBoT 62, Wiesbaden 2017.

Taggar-Cohen, Ada, Hittite Priesthood, THeth 26, Heidelberg 2006.

Taracha, Piotr, Religions of Second Millennium Anatolia, DBH 27, Wiesbaden 2009.

Tischler, Johann, Hethitische Texte in Transkription KUB 56 und KUB 57, DBH 49, Dresden 2016.

Ünal, Ahmet, Hititçe-Türkçe, Türkçe-Hititçe Büyük Sözlük, Ankara 2016.

Yoshida, Daisuke (1996). Untersuchungen zu den Sonnengottheiten bei den Hethitern, THeth 22, Heidelberg. 


\section{KISALTMALAR}

Ay. $\quad$ Arka yüz.

Öy. $\quad$ Ön yüz.

CHD The Hittite Dictionary of the Oriental Institute of the University of Chicago- Chicago 1980vd.

EDHIL Kloekhorst, A., Etymological Dicyionary of Hittite Inherited Lexicon 2008.

HEG Tischler, J., Hethitisches etymologisches Glossar, Innsbrucker 1977 vd.

HHw Tischler J., Hethitisches Handwörterbuch - Mit dem Wortschatz der Nachbarsprachen, 2., vermehrte und verbesserte Auflage, Innsbruck 2008.

MHH Ünal, A., Multilinguales Handwörterbuch des hethitischen, Hamburg 2007.

T. A. Tanrı/ça Adı. 